\title{
Double stranded RNA dependent protein kinase (PKR) and type 2 diabetes
}

\begin{abstract}
Type 2 diabetes greatly increases the risk for developing cardiovascular and metabolic disorders. Despite recent development in medical science, scientific understandings on the root mechanisms of type 2 diabetes are still not fully understood, and such insufficient understanding contributes to the relative lack of effective treatments for such diseases. Protein Kinase R (PKR) is a serine threonine kinase activated during various stress conditions. Activation of PKR can increase reactive oxygen species generation, inflammation and induce oxidative stress. In this review we discuss the potential role of PKR in type 2 diabetes, pathways activated by it and the interrelationship between pathways activated. Specific and effective inhibitors of PKR are being developed and can become potential treatment for type 2 diabetes and prevent many diseases.
\end{abstract}

Keywords: PKR, type 2 diabetes, inflammation, insulin resistance
Volume 2 Issue 2 - 2015

\author{
Arti Dhar,' Priyanka Reddy,' Audesh Bhat, ${ }^{2}$ \\ Indu Dhar ${ }^{3}$ \\ 'Department of Pharmacy, Birla Institute of Technology and \\ Sciences Pilani, India \\ ${ }^{2}$ Department of Microbiology \& Immunology, University of \\ Saskatchewan, Canada \\ ${ }^{3}$ Department of Pharmacology, University of Saskatchewan, \\ Canada
}

Correspondence: Arti Dhar, Department of Pharmacy, Birla Institute of Technology and Sciences Pilani, Jawahar Nagar, Shameerpet, Hyderabad, Andhra Pradesh 500078, India, Tel 04066303647,Email artidhar@hyderabad.bits-pilani.ac.in

Received: February 28, 2015 | Published: March 26, 2015

Abbreviations: PKR, protein kinase r; ER, endoplasmic reticulum; Nfkb, nuclear factor kappa-light-chain-enhancer of activated $b$ cells; IKK, inhibitory $\beta$ kinase; JNK, c-jun n-terminal kinases; Eif- $2 \alpha$, eukaryotic initiation factor $\alpha$

\section{Introduction}

Increasing type 2 diabetes and the associated hypertension and cardiovascular diseases in children and adults are major health issues globally with limited treatment options. The explosive increase in type 2 diabetes in the past 2-3 decades has been attributed to high dietary carbohydrates combined with a sedentary lifestyle. ${ }^{1,2}$ Such pathological conditions invoke stress related response in the body, one of which is activation of Protein Kinase R (PKR). RNA activated/ dependent protein kinase (PKR) is a serine threonine kinase that can directly couple to the metabolic pathway due to its catalytic activity and has a role in pathogen recognition. ${ }^{2} \mathrm{PKR}$ is activated by a number of signals, such as interferons, viral infection, high cholesterol diet, ${ }^{3}$ cytokines, pathogens, irradiation, heme limitation ${ }^{4-10}$ endoplasmic reticulum (ER) stress as well as mechanical stress. ${ }^{11}$ PKR contains two dsRNA binding domains, one at its N-terminal and the other at its C-terminal. ${ }^{4,5,12-14}$ In the presence of a pathogen, the double stranded RNA binds to the N-terminal of the PKR enzyme and leads to the phosphorylation of eukaryotic initiation factor (eIF2A). It activates eIF2AK2 (eukaryotic translation initiation factor 2-alpha kinase 2) which is coded by the eIF2AK2 gene., ${ }^{2,15}$ This increases phosphorylation of eIF2 and provide the kinase enzyme better access to its substrate. ${ }^{13,16}$ Increase in eIF $2 \alpha$ leads to the inhibition of translation thereby impeding further replication of the virus. In normal state, eIF2 combines with methionyl t-RNA and GTP, which is followed by its combination with the $40 \mathrm{~S}$ ribosomal subunit. This complex recognizes the start codon during translation. When the larger subunit is additionally combining with this complex, the GTPeIF2 complex is hydrolyzed to a GDP complex. During an infection, once eIF2 is phosphorylated to its eIF $2 \alpha$ form, the conversion of GTPeIF2 complex to GDP is inhibited. This results in blocking translation due to low GDP levels and thus prevents viral replication in cells. ${ }^{17,18}$
Type 2 diabetes is associated with elevated blood glucose levels, which in turn will affect plasma insulin levels. Components of the immune system are also changed in type 2 with the most apparent changes occurring in insulin sensitive tissues such as adipose tissue, liver, pancreatic islets as well as the vasculature and circulating leukocytes which in turn may lead to inflammation, insulin resistance, reduced insulin secretion, pancreatic $\beta$-cell apoptosis and tissue fibrosis. ${ }^{19}$ PKR is also implicated in inflammation and immune dysfunction through its regulation of mitogen-activated protein kinases, interferon regulatory factor 3 , nuclear factor $\mathrm{\kappa B}$ and apoptosis. $^{20}$

\section{Discussion}

It has been reported earlier that in PKR knockout $\left(\mathrm{Pkr}^{-/}\right)$mice, fasting plasma glucose is reduced while insulin action and insulininduced Akt phosphorylation is improved as compared to wild type control $\left(\mathrm{Pkr}^{+/+}\right)$mice. PKR is known to phosphorylate the regulatory subunit of PP2A, which then activates the catalytic subunit of PP2A inducing its phosphatase activity. ${ }^{3,21}$ Mice islet $\beta$-cells and insulinoma cell lines exposed to high glucose and proinflammatory cytokines showed significantly increased PKR activity associated with significantly inhibited cell proliferation by arresting cell cycle at G1 phase. PKR activation abolished the pro-proliferative effects of IGF-I by activating JNK and disrupting IRS1/PI3K/Akt signaling pathway. ${ }^{22}$ Inhibition of PKR reduces stress-induced JNK activation and IRS1 serine phosphorylation in vitro and in vivo. It has also been reported that treatment with PKR inhibitors, imoxin and 2-aminopurine reduced adipose tissue inflammation, improved insulin sensitivity, and improved glucose intolerance in obese (ob/ob) mice following the establishment of obesity and insulin resistance. ${ }^{3}$ PKR is known to directly target and modify the insulin receptor and thus inhibiting insulin action. It has been reported earlier that PKR induces the inhibitory phosphorylation of IRS at site Ser312 and activates the transcription factor, Foxo1, which in turn up-regulates the protein expression level of IRS2. Knockout of PKR $\left(\mathrm{Pkr}^{-/}\right)$in mice showed protection against insulin resistance and diabetes. ${ }^{3}$ 
Under stress condition, JNK negatively controls insulin signaling through serine phosphorylation of IRS1 instead of the normal tyrosine phosphorylation. JNK activation by PKR may also lead to serine phosphorylation of IRS1. ${ }^{23,24}$ Nakamura et al. ${ }^{3}$ tested this theory by taking $\mathrm{Pkr}$ knockout $\left(\mathrm{Pkr}^{-/}\right)$and wild type $\left(\mathrm{Pkr}^{+++}\right)$primary mouse embryonic fibroblast cells (MEFs) and exposing them to palmitic acid and thapsigargin. In Wild type $\left(\mathrm{Pkr}^{+/+}\right)$MEFs phosphorylation of IRS1 was observed whereas in PKR knockout $\left(\mathrm{Pkr}^{-/}\right)$MEF's, no IRS1 phosphorylation was observed. This proves that PKR is involved in the eventual phosphorylation of IRS1. ${ }^{3}$ Garcia et al. ${ }^{25}$ reported that when treating wild type $\left(\mathrm{Pkr}^{+/+}\right)$and PKR knockout $\left(\mathrm{Pkr}^{-/}\right)$MEFs with polyinosinic-polycytidylic (PolyI.C), a direct activator of PKR, IRS1 phosphorylation was only observed in wild type $\left(\mathrm{Pkr}^{+/+}\right)$MEFs whereas no phosphorylation was observed in PKR knockout $\left(\mathrm{Pkr}^{-/}\right)$ MEFs. ${ }^{25}$

Einarson et al. ${ }^{26}$ confirmed the interaction between PKR and IRS1 by Pull down assays, when a direct interaction was observed between IRS1 and PKR. ${ }^{26}$ Nakamura et al. ${ }^{3}$ reported that PKR causes the direct phosphorylation of the serine 307 residue of IRS1, using TNF- $\alpha$ (known to activate PKR) and TG on both wild type $\left(\mathrm{Pkr}^{+/+}\right)$ and PKR knockout $\left(\mathrm{Pkr}^{-/}\right)$MEFs and demonstrated the extent of IRS1 phosphorylation using a phospho specific antibody. It was found that the WT MEFs were able to show the excessive phosphorylation, unlike their counterparts. ${ }^{3}$ PKR plays an important role in insulin resistance as well. Nakamura et al. ${ }^{3}$ exposed wild type $\left(\mathrm{Pkr}^{+/+}\right)$and PKR knockout $\left(\mathrm{Pkr}^{/ /}\right)$mice to high fat diet and observed an increase in insulin induced Akt phosphorylation (Serine 473) in liver and adipose tissue of PKR knockout $\left(\mathrm{Pkr}^{-/}\right)$mice as compared to wild type $\left(\mathrm{Pkr}^{+/+}\right)$control mice. ${ }^{3}$ It has been reported previously that direct interactions of PKR occurs with the IKKb-NF- $\mathrm{KB}$ pathway. ${ }^{27} \mathrm{PKR}$ directly interacts with and modulates IRS, a critical molecule in insulin action, and has a major regulatory role over JNK activation. ${ }^{24}$ Thus pharmacologically targeting PKR may be an effective therapeutic strategy for the treatment of type 2 diabetes. The main limitation we have in this area is that the role of PKR in type 2 diabetes is still in the initial stages of investigation and more and more reports are coming out. Even then, these reports deal with the conditions obesity and diabetes as single entities and describe limited findings on the effects of PKR change in cultured cells and animal models. Apparently, it will take some time before an integrated picture of the role of PKR in the metabolic and cardiovascular disorders starts emerging.

\section{Conclusion and future prospects}

The present review indicates that PKR plays a crucial role in the pathogenesis of type 2 diabetes. Many important questions still remain to be addressed. Current understanding on the inflammatory mechanisms of metabolic syndrome and related disorders are still in its primitive stage. We anticipate in near future eventually these findings will be translated into novel and effective treatments/preventions against diabetes and related diseases.

\section{Acknowledgements}

None.

\section{Conflict of interest}

Author declares that there is no conflict of interest.

\section{References}

1. Olokoba AB, Obateru OA, Olokoba LB. Type 2 Diabetes Mellitus: A Review of Current Trends. Oman Med J. 2012;27(4):269-273.

2. Samuel CE. The eIF-2 alpha protein kinases, regulators of translation in eukaryotes from yeasts to humans. $J$ Biol Chem. 1993;268(11):7603-7606

3. Nakamura T, Furuhashi M, Li P, et al. Double-stranded RNA-dependent protein kinase links pathogen sensing with stress and metabolic homeostasis. Cell. 2010;140(3):338-348.

4. Clemens MJ. Protein Kinases That Phosphorylate eIF2 and eIF2B, and Their Role in Eukaryotic Cell Translational Control. Cold Spring Harbor Monograph Archive. 1996;30:139-172.

5. Proud CG. PKR: a new name and new roles. Trends Biochem sci. 1995;20(6):241-246.

6. Kaufman RJ. Stress signaling from the lumen of the endoplasmic reticulum: coordination of gene transcriptional and translational controls. Genes Dev. 1999;13(10):1211-1233.

7. Brostrom CO, Brostrom MA. Regulation of translational initiation during cellular responses to stress. Prog Nucleic Acid Res Mol Biol. 1998;58:79-125.

8. Gale M, Katze MG. Molecular mechanisms of interferon resistance mediated by viral-directed inhibition of PKR, the interferon-induced protein kinase. Pharmacol Ther. 1998;78(1):29-46.

9. Wek RC. elF-2 kinases: regulators of general and gene-specific translation initiation. Trends in biochemical science. 1994;19(11):491-496.

10. Hinnebusch AG. Translational regulation of yeast GCN4. A window on factors that control initiator-tRNA binding to the ribosome. $J$ Biol Chem. 1997;272(35):21661-21664.

11. Ron D, Walter P. Signal integration in the endoplasmic reticulum unfolded protein response. Nat Rev Mol Cell Biol. 2007;8(7):519-529.

12. Williams BR. PKR; a sentinel kinase for cellular stress. Oncogene. 1991;18(545):6112-6120.

13. Wu S, Kumar KU, Kaufman RJ. Identification and requirement of three ribosome binding domains in dsRNA-dependent protein kinase (PKR). Biochemistry. 1998;37(39):13816-13826.

14. McCormack SJ, Ortega LG, Doohan JP, et al. Mechanism of interferon action motif I of the interferon-induced, RNA-dependent protein kinase (PKR) is sufficient to mediate RNA-binding activity. Virology. 1994;198(1):92-99.

15. Feng G, Chong K, Kumar A, et al. Identification of double-stranded RNA-binding domains in the interferon-induced double-stranded RNA-activated p68 kinase. Proc Natl Acad Sci USA. 1992;89(12):5447-5451.

16. Zhu S, Romano PR, Wek RC. Ribosome targeting of PKR is mediated by two double-stranded RNA-binding domains and facilitates in vivo phosphorylation of eukaryotic initiation factor-2. J Biol Chem. 1997;272 (22):14434-14441.

17. Hershey JW, Merrick WC. Pathway and mechanism of initiation of protein synthesis. Cold Spring Harbor Monograph Archive. 2000;39:33-88.

18. Kaufman RJ. Double-stranded RNA-activated protein kinase mediates virus-induced apoptosis: a new role for an old actor. Proc Natl Acad Sci USA. 1999;96(21):11693-11695.

19. Donath MY, Shoelson SE. Type 2 diabetes as an inflammatory disease. Nat Rev Immunol . 2011;11(2):98-107. 
20. Kang R, Tang D. PKR-dependent inflammatory signals. Sci Signal 2012;5(247):pe47.

21. Goh KC, DeVeer MJ, Williams BR. The protein kinase PKR is required for p38 MAPK activation and the innate immune response to bacteria endotoxin. EMBO J. 2000;19(16):4292-4297.

22. Chen SS, Jiang T, Wang Y, et al. Activation of double-stranded RNA-dependent protein kinase inhibits proliferation of pancreatic $\beta$-cells. Biochem Biophys Res Commun. 2014;443(3):814-820.

23. Yang X, Nath A, Opperman MJ, et al. The Double-stranded RNA-dependent Protein Kinase Differentially Regulates Insulin Receptor Substrates 1 and 2 in HepG2 Cells. Mol Biol Cell. 2010;21(19):3449-3458.
24. Dearth RK, Cui X, Kim HJ, et al. Mammary tumorigenesis and metastasis caused by overexpression of insulin receptor substrate 1 (IRS-1) or IRS-2. Mol cell biol. 2006;26(24):9302-9314.

25. Garcia MA, Gil J, Ventoso I, et al. Impact of protein kinase PKR in cell biology: from antiviral to antiproliferative action. Microbiol Mol Biol Rev. 2006;70(4):1032-1060.

26. Einarson MB, Pugacheva EN, Orlinick JR. Identification of protein-protein interactions with glutathione-S-transferase (GST) fusion proteins. Cold Spring Harbor Protocols. 2007.

27. Bonnet MC, Weil R, Dam E, et al. PKR stimulates NF-kappaB irrespective of its kinase function by interacting with the IkappaB kinase complex. Mol Cell Biol. 2000;20(13):4532-4542. 